quotient ist weiterhin die Frage zu diskutieren, ob diese Abweichungen durch einen dritten unbekannten Faktor bei urämischen Patienten hervorgerufen werden (22). Diese Möglichkeit läßt sich durch unsere Befunde nicht sicher ausschließen, obgleich die oben aufgeführten experimentellen Untersuchungen eher daran denken lassen, daß die Änderungen des Natrium- und Kaliumquotienten durch den $\mathrm{pH}$-Wert beeinflußt werden.
Für die Behandlung der chronischen Niereninsuffizienz bzw. Urämie ergibt sich aus unseren Befunden, daß durch Einsatz der Hämodialyse sowohl Veränderungen des Säure-Basen- als auch des Elektrolythaushaltes behoben werden können. Diese Normalisierung ist wiederum die Voraussetzung dafür, $\mathrm{da} B$ weitere folgenschwere Störungen des Zellstoffwechsels vermieden werden bzw. eine normale Organfunktion wiederhergestellt wird.

\title{
Literatur
}

1. Gessler, U., Verh. Dtsch. Ges. inn. Med. 66, 870 (1960). 2.Gessler, U., Klin. Wschr. 39, 232 (1961). - 3. HäNZE, S., Klin. Wschr. 38, 769 (1960). - 4. RIECKER, G., Klin. Wschr. 35, 1158 (1957). - 5. RIECKER, G., Klin. Wschr. 41, 184 (1963). - 6. BURCK, H. C., Zschr. exper. Med. 144, 93 (1967). - 7. Wilbrandt, W., J. Pharmacy Pharmacol. London 11, 65 (1959). - 8. WILBRANDt, W., Klin. Wschr. 41, 138 (1963). - 9. ZuMKLEY, H., Zschr. Kreisl. forsch. 56, 678 (1967). - 10. GleichmanN, K., H. v. STUCKRAD und M. ZindLER, Zschr. exper. Med. 139, 255 (1965). 11. Sommerkamp, H. und K. Bomke, Klin. Wschr. 42, 392 (1964). 12. Zumklex, H. und H. Losse, Gastroenterologia Basel 104, 136 (1965). - 13. Losse, H., H. WehMeYer und F. WesseLs,
Klin. Wschr. 38, 393 (1960). - 14. Losse, H., H. ZUMKLEY und H. WeHMEYER, Zschr. Kreisl.forsch. 51, 752 (1962). - 15. BRESCIANI, F., F. Auricchio und C. Fiore: Nature, London 196, 186 (1962). - 16. Konsek, I. und C. Bishop, Proc. Soc. exp. Biol. Med. N.Y. 110, 813 (1962). - 17. WILBRAND̈r, W., Med. Klin. 50, 2085 (1962). - 18. Gessler, U. und W. Caliebe, Zschr. exper. Med. 134, 139 (1961). - 19. Gessier, U., Internist 3, 672 (1962). 20. Scribner, B. H., K. Fremont-Smith und J. M. Bưrnell, J. Clin. Invest. 34, 1276 (1955). - 21. Bubnoff, v. M. und G. RIECKER, Klin. Wschr. 39, 724 (1961). - 22. Sarre, H., U. Gessler und V. Heinze, Internist 10, 446 (1965).
Dr. H. Zumkley 44 Münster (Westf.) Westring 3

\section{Eine bewährte Methode zur routinemäßigen Bestimmung des proteingebundenen Jods (PBI)}

\author{
Von A. UETTWILLER \\ Aus den Laboratorien (Leiter: Dr. M. Keller) der Universitäts-Fraulenklinik Basel \\ (Direktor: Prof. Dr. Th. Koller)
}

(Eingegangen am 17. Dezember 1967)

\begin{abstract}
Es wird eine zuverlässige Routinemethode zur Bestimmung von PBI im Blutplasma beschrieben. Die oxydative Aufschlußzeit konnte wesentlich verkürzt werden. Eine Vereinfachung der Meßbedingungen wurde durch Unterbrechung der katalytischen Wirkung des Jods auf die Reaktion: $\mathrm{Ce}^{4+} \longrightarrow \mathrm{Ce}^{3+}$ mittels $\mathrm{Zusatz}$ von $\mathrm{Hg}^{2+}$-Ionen erreicht.
\end{abstract}

A reliable, routine method is described for the measurement of PBI in blood plasma. The time for oxidative cleavage was greatly reduced. The determination was simplified by adding $\mathrm{Hg}^{2+}$ ions, which stop the catalytic action of the iodine in the reaction: $\mathrm{Ce}^{4+} \longrightarrow \longrightarrow \mathrm{Ce}^{3+}$.

Es wird im folgenden eine Methode zur PBI-Bestimmung im Blutplasma beschrieben, die sowohl als Makro-' ( $1 \mathrm{~m} l$ Plasma) als auch Semimikro- $(200 \mu l)$ Methode in unserem Laboratorium seit einiger Zeit Anwendung findet. Das Verfahren basiert auf den Angaben von FARELL und Richmond (1) sowie denjenigen von FriedmanN (2), welche die nasse Veraschung von jodhaltigen Proteinen beschrieben.

Unsere wichtige Modifikation verkürzt die oxydative Aufschlußzeit von $4 \frac{1}{2}$ Stdn. auf $1 \mathrm{Std}$. Weiter werden die Meßbedingungen wesentlich vereinfacht, indem die katalytische Wirkung von $\mathrm{J}_{2}$ auf die Reaktion:

$$
\mathrm{Ce}^{+4}+\mathrm{As}^{+3} \longrightarrow \mathrm{Ce}^{+3}+\mathrm{As}^{+5}
$$

mit $\mathrm{Hg}^{+2}$-Ionen gestoppt wird. Die țechnischen Schwierigkeiten, welche die meisten bekannten Plasmajodbestimmungsmethoden aufweisen, konnten dadurch weitgehend behoben werden.

\section{Prinzip}

Das mit $\mathrm{HClO}_{4}$ gefällte Eiweiß wird mit einem Chlorsäure-Dichromat-Gemisch oxydativ aufgeschlossen:

$$
\mathrm{R}-\mathrm{J} \underset{\mathrm{HClO}_{2}}{\stackrel{\mathrm{K}_{2} \mathrm{Cr}_{2} \mathrm{O}_{7}}{\longrightarrow}} \mathrm{R}-+\mathrm{JO}_{\mathbf{3}}^{-}
$$


Die Reduktion von $\mathrm{JO}_{\overline{3}}^{-}$mit $\mathrm{As}^{3+}$ erfolgt nach:

$$
\mathrm{JO}_{3}^{-}+3 \mathrm{As}^{3+}+6 \mathrm{H}^{+} \longrightarrow \mathrm{J}^{-}+3 \mathrm{As}^{5+}+3 \mathrm{H}_{2} \mathrm{O}
$$

Die Katalyse der Reaktion erfolgt nach:

$$
\mathrm{Ce}^{4+}+\mathrm{As}^{3+} \stackrel{\left[\mathrm{J}_{2}\right]}{\longrightarrow} \mathrm{Ce}^{3+}+\mathrm{As}^{5+}
$$

und kommt vermutlich so zustande:

$$
\begin{aligned}
& 2 \mathrm{~J}^{-}+2 \mathrm{Ce}^{4+} \longrightarrow \mathrm{J}_{2}+2 \mathrm{Ce}^{3+} \\
& \mathrm{J}_{2}+\mathrm{HOH} \stackrel{[\mathrm{Cl}-]}{\longrightarrow} \mathrm{JOH}+\mathrm{J}^{-}+\mathrm{H}^{+} \\
& \mathrm{JOH}+\mathrm{As}^{3+} \longrightarrow \mathrm{J}^{-}+\mathrm{As}^{5+}
\end{aligned}
$$

Die beschleunigte Reduktion von $\mathrm{Ce}^{4+} \mathrm{zu} \mathrm{Ce}^{3+}$ kann nun nach der notwendigen Reaktionszeit ${ }^{1}$ ) durch Zugabe von $\mathrm{Hg}^{2+}$-Ionen unterbrochen werden:

$$
4 \mathrm{~J}^{-}+\mathrm{Hg}^{2+} \longrightarrow\left[\mathrm{HgJ}_{4}\right]^{2-}
$$

Kinetische Untersuchungen bestätigen in anschaulicher Weise diesen Mechanismus (Abb. 1).

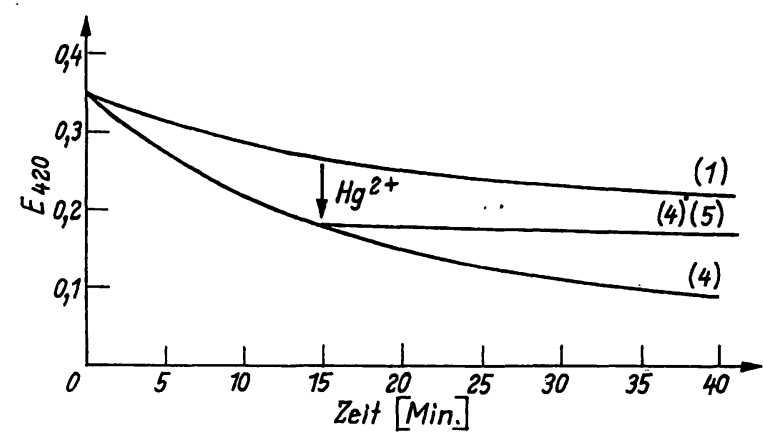

Abb. 1

Spektrophotometrische Untersuchungen des Reaktionsverlaufs $\mathrm{Ce}^{\mathrm{st}}+\mathrm{As}^{3+} \stackrel{\left[\mathrm{J}_{2}\right]}{\longrightarrow} \mathrm{Ce}^{3+}+\mathrm{As}^{3+}$

Die Kurven (1) und (4) zeigen die Extinktionsabnahme in Funktion der Zeit (Farbintensitätsabnahme von $\mathrm{Ce}^{s+}$ gemessen bei $\lambda$ (konstant) $=420 \mathrm{~nm}$ ) der Reaktion [1] und [4] (siehe Text). Die nahezu horizontal verlaufende Kurve (4) (5) zeigt den Einfluß der nach 15 Min. zugesetzten $\mathrm{Hg}^{2+}$-Ionen auf die durch $\mathrm{J}_{2}$ katalysierte $\mathrm{Re}$ aktion

\section{Methodik}

\section{Apparate}

Spektrophotometer Unicam SP 600 mit automatischer Durchflußküvette $(\mathrm{d}=1 \mathrm{~cm})$.

Elektrischer Aluminiumheizblock mit regulierbarer Temperatureinstellung. Zentrifuge (3000 U./Min.) Zentrifugengläser $(40 \mathrm{ml}$ Inhalt).

\section{Reagenzien}

Bidest. Wasser für sämtliche Lösungen, Verdünnungen und Reagenzien!

Perchlorsäure 1,1N (Merck).

Chlorsäure $\left(\mathrm{HClO}_{3}\right): 92 \mathrm{~g} \mathrm{KClO}_{3}$ (aus Wasser umkristallisiert) in $250 \mathrm{ml}$ kochendem Wasser lösen. Zur heißen Lösung vorsichtig $75 \mathrm{ml} \mathrm{HClO}_{4}(70-72 \%)$ unter stetigem Rühren zugeben. Uber Nacht im Kühlschrank stehenlassen und vor Gebrauch schnell durch eine Glasfilternutsche abfiltrieren. Es ist wichtig, daß die Chlorsäure vor dem PBI-Bestimmungstag immer frisch hergestellt werden muß.

1) Versuche ergaben, daß für einen PBI-Bereich von 0,5-15 $\mu \mathrm{g}$ / $100 \mathrm{ml}$ eine Reaktionszeit von $15 \mathrm{Min}$. sehr geeignet ist.
$\mathrm{K}_{2} \mathrm{Cr}_{2} \mathrm{O}_{7}$-Lösung (10-2 $\left.\mathrm{M}\right): 2,94 \mathrm{~g} \mathrm{~K}_{2} \mathrm{Cr}_{2} \mathrm{O}_{7}$ ad $1000 \mathrm{~m} /$ Wasser. $\mathrm{As}^{3+}$-Lösung (10-1 $\left.\mathrm{M}\right): 20 \mathrm{~g} \mathrm{As}_{2} \mathrm{O}_{3}$ in $83 \mathrm{ml} 2,5 \mathrm{~N} \mathrm{NaOH}$ lösen. Dann Zugabe von etwa $700 \mathrm{~m} /$ Wasser und Zusatz von $83 \mathrm{~m} / \mathrm{konz}$. $\mathrm{H}_{2} \mathrm{SO}_{4}$. Abkühlen und mit Wasser auf $1000 \mathrm{ml}$ auffüllen.

$\mathrm{As}^{3+}$-Lösung $\left(10^{-2} \mathrm{M}\right): 100 \mathrm{ml} \operatorname{der} 10^{-1} \mathbf{M ~ A s}^{3+}$-Lösung mit $20 \mathrm{ml}$ konz. $\mathrm{HCl}$ und anschließend mit $75 \mathrm{~m} /$ konz. $\mathrm{H}_{2} \mathrm{SO}_{4}$ versetzen. Mit Wasser auf $1000 \mathrm{~m} l$ auffüllen.

$\mathrm{Ce}^{4+}$-Lösung $(0,5 \mathrm{M}): 14 \mathrm{ml}$ konz. $\mathrm{H}_{2} \mathrm{SO}_{4}$ werden unter Rühren zu $137 \mathrm{~g}\left(\mathrm{NH}_{4}\right)_{2} \mathrm{Ce}\left(\mathrm{NO}_{3}\right)_{6}$ gegeben. Zusatz von $50 \mathrm{ml}$ Wasser bis vollständige Lösung erfolgt und auffüllen auf $500 \mathrm{~m} / \mathrm{mit}$ Wasser. $\mathrm{Ce}^{4+}$-Lösung $\left(10^{-2} \mathrm{M}\right): 10 \mathrm{ml}$ der $0,5 \mathrm{M} C \mathrm{Ce}^{4+}$-Lösung mit $42 \mathrm{ml}$ konz. $\mathrm{H}_{2} \mathrm{SO}_{4}$ versetzen. Anschließend mit Wasser auf $500 \mathrm{~m} /$ auffüllen.

$\mathrm{Hg}^{2+}$-Lösung (15\% W/V): $150 \mathrm{~g} \mathrm{Hg}\left(\mathrm{CH}_{3} \mathrm{COO}\right)_{2}$ ad $1000 \mathrm{~m} /$ 2proz. Essigsäure. Die trübe Lösung wird filtriert.

Sämtliche Gebrauchslösungen werden im Eisschrank aufbewahrt. Sie sind stabil. Die einzige Ausnahme bildet die Chlorsäure, die wie oben schon erwähnt immer frisch zubereitet werden muß. Der angegebene Chlorsäureansatz reicht für 20 Doppelbestimmungen.

\section{Bemerkungen}

Die Glasgeräte und die Küvetten müssen durch Einlegen in Chromschwefelsäure gereinigt werden. Nach mehrmaligem Spülen mit Leitungswasser zweimal mit bidest. Wasser nachspülen. Das Veraschen der Proben muß unter einem Abzug erfolgen. Die Bestimmungen werden immer im Doppel ausgeführt. Man teilt die Proben in zwei gleiche Gruppen auf. Mit jeder Gruppe laufen ein Leerwert und zwei Standards mit. Erst nach erfolgter Ablesung der ersten Gruppe wird das $\mathrm{Ce}^{4+}+$ Reagenz zu den Proben der zweiten Gruppe gegeben.

\section{Präzision der Methode}

Makroverfahren: $\quad \mathrm{n}=50, \mathrm{~s}= \pm 0,18 \mu \mathrm{g} / 100 \mathrm{ml}$,

$$
\text { V. K. }=5,4 \% \text {. }
$$

Semimikroverfahren: $\mathrm{n}=20, \mathrm{~s}= \pm 0,23 \mu \mathrm{g} / 100 \mathrm{ml}$,

$$
\text { V. K. }=7,8 \% \text {. }
$$

Zeitaufwand

In vier Stunden können 20 Doppelanalysen oder 40 Einzelanalysen durchgeführt werden.

ARBEITSVORSCHRIFT: MAKROMETHODE

\begin{tabular}{lccc}
\hline Reagenzien & $\begin{array}{c}\text { Reagenzien- } \\
\text { Leerwert }\end{array}$ & Standard & Analyse \\
\hline $\begin{array}{l}\text { Hylandserum } \\
\text { Plasma } \\
\mathrm{HClO}_{4} 1,1 \mathrm{~N}^{*}\end{array}$ & - & $1 \overline{\mathrm{ml}}$ & $1 \overline{\mathrm{m} l}$ \\
\hline
\end{tabular}

* Die $\mathrm{HC1O}$, wird tropfenweise unter ständigem Rühren zugegeben. Zentrifugieren, überstehende Lösung dekantieren.

\begin{tabular}{lccc}
\hline $\mathrm{K}_{2} \mathrm{Cr}_{2} \mathrm{O}_{7}\left(10^{-2} \mathrm{M}\right)$ & $\begin{array}{c}0,4 \mathrm{ml} \\
\mathrm{HClO}_{3}\end{array}$ & $\begin{array}{c}0,4 \mathrm{ml} \\
5 \mathrm{ml}\end{array}$ & $\begin{array}{c}0,4 \mathrm{ml} \\
5 \mathrm{ml}\end{array}$ \\
\hline
\end{tabular}

40 Min. bei $125^{\circ}-130^{\circ}$ digerieren (Heizblock vorher auf Temp. stellen). Temp. auf $160^{\circ}$ erhöhen. Bei blau-grün-Färbung des Rückstandes 1 Tropfen $\mathrm{HClO}_{3}$ zugeben und

\begin{tabular}{llll}
\hline $\mathrm{As}^{8+}\left(10^{2} \mathrm{M}\right)$ & $5 \mathrm{ml}$ & $5 \mathrm{ml}$ & $5 \mathrm{ml}$ \\
$\mathrm{H}_{2} \mathrm{O}$-bidest* & $7 \mathrm{ml}$ & $7 \mathrm{ml}$ & $7 \mathrm{ml}$ \\
\hline
\end{tabular}

* Unter Schütteln zugeben, bis der Rückstand gelöst ist.

\begin{tabular}{llll}
\hline $\mathrm{Ce}^{a+}\left(10^{-2} \mathrm{M}\right)^{*}$ & $1 \mathrm{ml}$ & $1 \mathrm{ml}$ & $1 \mathrm{ml}$ \\
\hline
\end{tabular}

* In 15 Sek. Zeitabständen zwischen jeder Probe zugeben. Genau 15 Min. reagieren lassen.

$\mathrm{Hg}^{\mathrm{a}+15 \% *} 1 \mathrm{ml} \quad 1 \mathrm{ml} \quad 1 \mathrm{ml}$

* In 15 Sek. Zeitabständen zwischen jeder Probe zugeben. Ablesen bei $420 \mathrm{~nm}$ gegen $\mathrm{H}, \mathrm{O}(\mathrm{d}=1 \mathrm{~cm}$ Küvette). 
ARBEITSVORSCHRIFT: SEMIMIKROMETHODE

\begin{tabular}{lccc}
\hline Reagenzien & $\begin{array}{c}\text { Reagenzien- } \\
\text { Leerwert }\end{array}$ & Standard & Analyse \\
\hline $\begin{array}{l}\text { Hylandserum } \\
\text { Plasma } \\
\text { HCIO } 1,1 \mathrm{~N}^{*}\end{array}$ & $=$ & $200 \mu l$ & $200 \mu l$ \\
\hline
\end{tabular}

Zentrifugen und dekantieren.

\begin{tabular}{llll}
\hline $\mathrm{K}_{2} \mathrm{Cr}_{2} \mathrm{O}_{7}\left(10^{-2} \mathrm{M}\right)$ & $100 \mu l$ & $100 \mu l$ & $100 \mu l$ \\
$\mathrm{HClO}_{3}$ & $1,5 \mathrm{ml}$ & $1,5 \mathrm{ml}$ & $1,5 \mathrm{ml}$ \\
\hline
\end{tabular}

20 Min. bei $125^{\circ}-130^{\circ}$ digerieren (Heizblock vorher auf Temp. stellen). Temp. auf $160^{\circ}$ erhöhen. Bei farblos-blau-Färbung des Rückstandes $20 \mu l \mathrm{HClO}_{3}$ zugeben und wegnehmen (Reagenzienleerwert wird rot). - Abkühlen lassen.

\begin{tabular}{lccc}
\hline $\mathrm{As}^{3+}\left(10^{-2} \mathrm{M}\right)$ & $500 \mu l$ & $500 \mu l$ & $500 \mu \mathrm{l}$ \\
$\mathrm{H}_{2} \mathrm{O}$ bidest* & $2 \mathrm{ml}$ & $2 \mathrm{ml}$ & $2 \mathrm{ml}$ \\
\hline
\end{tabular}

Rückstand lösen.

\begin{tabular}{llll}
\hline $\mathrm{Ce}^{4+}\left(10^{-2} \mathrm{M}\right)^{*}$ & $200 \mu l$ & $200 \mu l$ & $200 \mu l$ \\
\hline
\end{tabular}

In 15 Sek. Zeitabständen zugeben.

\begin{tabular}{llll}
\hline $\mathrm{Hg}^{2+} 15 \% *$ & $200 \mu l$ & $200 \mu l$ & $200 \mu l$ \\
\hline
\end{tabular}

Nach 15 Min. in 15 Sek. Zeitabständen zugeben.

Ablesen bei $420 \mathrm{~nm}$ gegen $\mathrm{H}_{2} \mathrm{O}$ (d $=1 \mathrm{~cm}$ Küvette).

* Arbeitsverfahren siehe Makromethode.

Die Auswertung der Resultate erfolgt graphisch. Für jede Meßgruppe wird eine Eichkurve erstellt (Extinktion des Leerwerts und der Standards gegen Äquivalent PBI $\mu \mathrm{g} / 100 \mathrm{~m} l$ ).

\section{Resultate}

An Hand von über 1500 PBI-Bestimmungen lassen sich folgende Aussagen machen: Der Mittelwert kann aus der graphischen Darstellung (Abb. 2) entnommen werden. Er liegt mit großer Wahrscheinlichkeit bei $5 \mu \mathrm{g} / 100 \mathrm{~m} l$. Der deutlich begrenzte Normalbereich liegt $z$ wischen den Werten 3,4 bis $7,2 \mu \mathrm{g} / 100 \mathrm{ml}$.

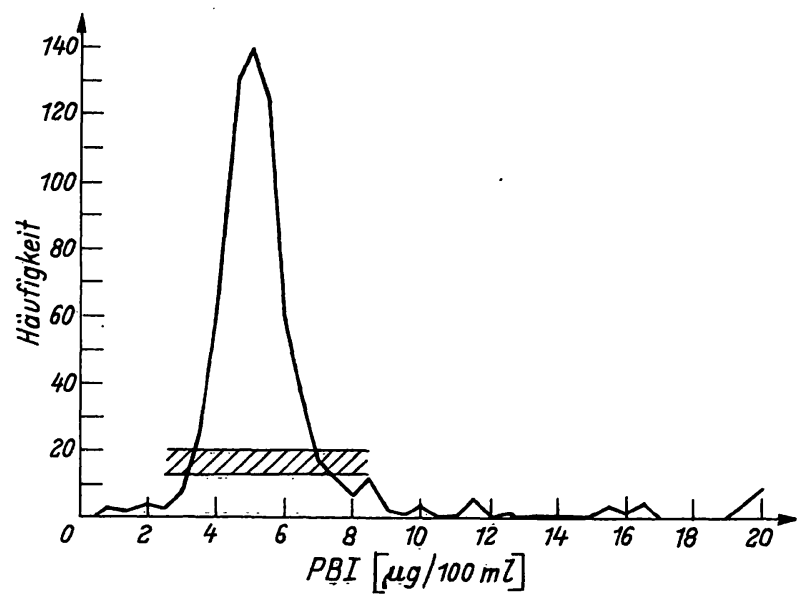

Abb. 2

Werteverteilung von PBI (1000 Fälle). Mittelwert $5 \mu \mathrm{g} / 100 \mathrm{ml}$. Normalbereich $3,4-7,2 \mu \mathrm{g} / 100 \mathrm{ml}$

\section{Literatur}

1. FARELL, L. P. und M. H. Rrchmond, Clin. chim. Acta (Amsterdam) 6, 620 (1961). - 2. Friedman, H. S., Clin. chim. Acta (Amsterdam 7, 111 (1962).

Dr. A. Uettwiller CH 4056 Basel, Schweiz Schanzenstr. 46

\section{BUCHBESPRECHUNGEN}

Praktikum der Physiologischen Chemie. Von SIEgmundSCHÜTTE-KöRBER. XVI, 287 Seiten m. 49 Abb. br. DM 19,80. Verlag Walter de Gruyter \& Co., Berlin 1968.

In 6 großen - nach Methoden geordneten - Gruppen ist eine Fülle von Versuchen aufgeführt, die, bei qualitativen Nachweisen beginnend, über die Maßanalytik und Potentiometrie zu optischen und chronometrischen Methoden führen und schließlich dünnschichtchromatographische und elektrophoretische Trennungen einschließen. Jeder Art von Versuchen (mit Beschreibung) sind kurze theoretische Erläuterungen vorangestellt, die dort jeweils ausführlicher gehalten sind, wo entsprechende Erklärungen in den meisten Lehrbüchern der Physiologischen Chemie vermißt werden.

Eine Reihe von "Schnelltests“ und enzymatischen Substratnachweisen haben viele früher übliche Farbreaktionen verdrängt. So wird auch hier eine größere Anzahl derartiger Verfahren besprochen wohl im Hinblick darauf, daß der weitaus größte Teil der Kursteilnehmer später einmal praktische Medizin betreiben wird.

Im Mittelpunkt steht jedoch die Biochemie der Enzyme: die Erkennung ihrer Eigenschaften, die Bestimmung von Aktivitäten, Konzentrationen usw., wodurch auch an den gut vorgebildeten und biochemisch interessierten Studenten höhere Ansprüche gestellt werden. Dazu sind natürlich $z$. T. recht aufwendige Versuchsanordnungen notwendig. Hier wird aber durch das am Ende des Buches angegebene Verzeichnis der für die einzelnen Kurstage benötigten Lösungen, Reagenzien und Geräte eine wesentliche Erleichterung zur Vorbereitung des Praktikums gegeben.
Es dürfte wohl unmöglich sein, bei der dem Studenten der Medizin heute für die Physiologische Chemie zur Verfügung stehenden Zeit und den augenblicklich noch sehr großen Studentenzahlen, alle hier angegebenen Versuche durchzuführen. Das ist wohl auch von den Autoren nicht intendiert. Sie schlagen im Vorwort selbst eine gut durchführbare Auswahl vor.

Es ist außerordentlich dankenswert, daß die Autoren ihre langen Erfahrungen, die sie bei der Durchführung des physiol.-chem. Praktikums an der Freien Universität Berlin sammelten, in dieser umfassenden, didaktisch hervorragenden Form, mit vielen sehr klaren Abbildungen und Diàgrammen ausgestattet, an alle Interessierten weitergeben.

Es bleibt zu wünschen, daß man sich dort, wo Praktika in der Physiologischen Chemie abgehalten werden, dieses Buches bedient und entsprechend der apparativen Ausstattung eine möglichst vielseitige Auswahl der Versuchẹ trifft.

H. Debuch, Köln

Cross Electrophoresis. Its Principle and Applications. Von S. Nakamura. IX, 194 S., zahlr. Abb. und Tab., Dfl. 47,50.

Igaku Shoin Ltd., Tokyo-Elsevier Publishing Comp., AmsterdamLondon-New York (1966).

Unter dem Titel: „Ein einfaches elektrophoretisches Verfahren zum Nachweis lockerer Additionsverbindungen" beschrieben W. GrASSMANN und L. HüBNER 1953 in den Naturwissenschaften (40, 272) die Modifikation einer Elektrophorese-Technik, die von den Autoren des vorliegenden Büches weiter vervollkommnet und für zahlreiche biochemische Problemstellungen angewandt wurde. Das als „Cross Electrophoresis“" bezeichnete 